\title{
RASPBERRY PI BASED MICROSCOPE CAMERA WITH WIRELESS FUNCTIONALITY
}

\author{
A. C. GHEORGHE \\ Department of Electronics, Telecommunications and Energy Engineering, Valahia University of Targoviste \\ E-mail: gheorghe_andrei89@yahoo.com
}

\begin{abstract}
The paper proposes the development of a wireless video camera that is used to capture images from any microscope, and is universally compatible with the standard $C$-Mount. The purpose behind developing this camera is to offer an economical alternative to the current high-priced microscopy camera without compromising performance. In addition this camera has the technology to work wirelessly.
\end{abstract}

Keywords: wireless. video camera, performance, economical

\section{INTRODUCTION}

The paper proposes the development of a wireless video camera that is used to capture images from any microscope and it is universally compatible with the standard C-Mount. The purpose behind developing this camera is to offer an economical alternative to the current high-priced microscopy camera without compromising performance. In addition this camera has the technology to work wirelessly. The camera is made out of four major components [1], [2]:

- Raspberry Pi Zero W that is used to save all the data captured form the sensor, either to save or to send the data over IP (Internet Protocol) or to a web interface. The Pi Zero is very small in size and can be fitted in a small enclosure.

- Raspicam v2 is used as the actual camera because it has an IMX219 Sony sensor that has a good quality price and offers good performance.

- For the optics we used and optic adapter with a fixed focal length, the adapter is a FMA037 that we can find on the market at a cheap price and it has a $0.37 \mathrm{X}$ magnification, is fit to support a $1 / 4 "-1 / 3$ " sensor and has the eye tube standard c-mount to Dia. $23.2 \mathrm{~mm}$.

- A LiPo battery that offers the power that we need to use the camera for a few hours, the battery gives $5 \mathrm{v}$ and has the capacity of $5500 \mathrm{mAh}$.

\subsection{Raspberry Pi Zero W}

The Raspberry Pi Zero W development board is very unique in size as can be seen in Figure 1 and gives us a way to connect with the Raspicam v.2 trough the CSI (Camera Serial Interface) port. The Raspberry Pi Zero W (Figure 2) has a single-core CPU at $1 \mathrm{GHz}$ with $512 \mathrm{MB}$ of RAM, $802.11 \mathrm{~b} / \mathrm{g} / \mathrm{n}$ wireless LAN and the CSI port. The operating system on the raspberry is a modified Linux distribution specially made for the $\mathrm{Pi}$ named Raspbian. The Raspberry Pi acts like a server that send over IP the images obtained from the Sony sensor or saves them on the internal storage that is about $16 \mathrm{~GB}$ of space, this method is used for later downloading from the internal storage [3].

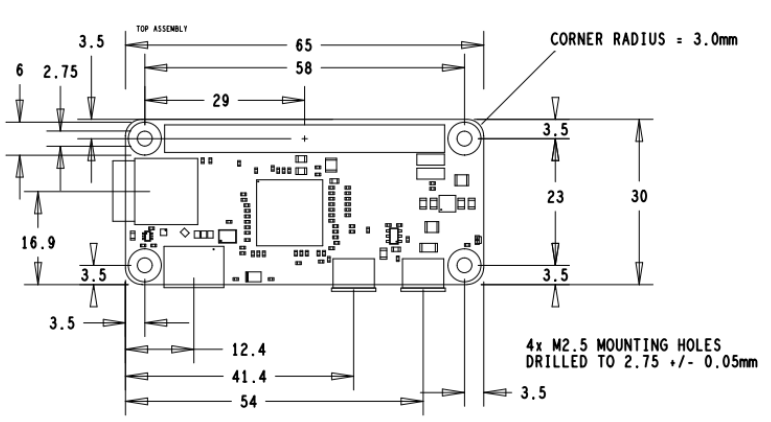

Figure 1. Mechanical schematic

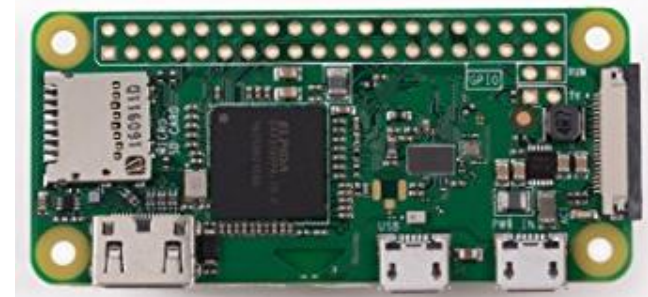

Figure 2. Raspberry Pi Zero W development board

\subsection{Raspcam v.2}

The camera sensor used (Sony IMX219) has a good quality price and is specially design to be used with the Raspberry Pi trough the CSI port. The optic sensor has a diagonal size of $4.60 \mathrm{~mm}$, type $1 / 4.0$ " with a $8 \mathrm{MPX}$ (Mega Pixels) that offers video modes of $1080 \mathrm{p}$ (1920x1080) at 30 FPS (Frames Per Second), 720p $(1024 \times 720)$ at 60 FPS and 480p (640x480) at 60/90 FPS. The Linux software integration is made trough the V4L2 driver, the pixel size is $1.12 \mu \mathrm{m} \times 1.12 \mu \mathrm{m}$ and the supported resolution for pictures is $3280 \times 2464$ pixels. In Figure 3 we can see the sensor already installed in the camera enclosure [4], [5].

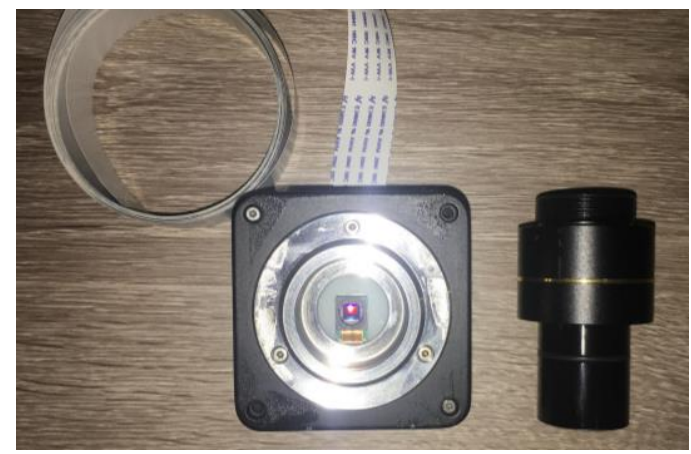

Figure 3. Optical sensor installed in the optical enclosure 
In Figure 4 we can see the block diagram of the Sony IMX219 sensor.

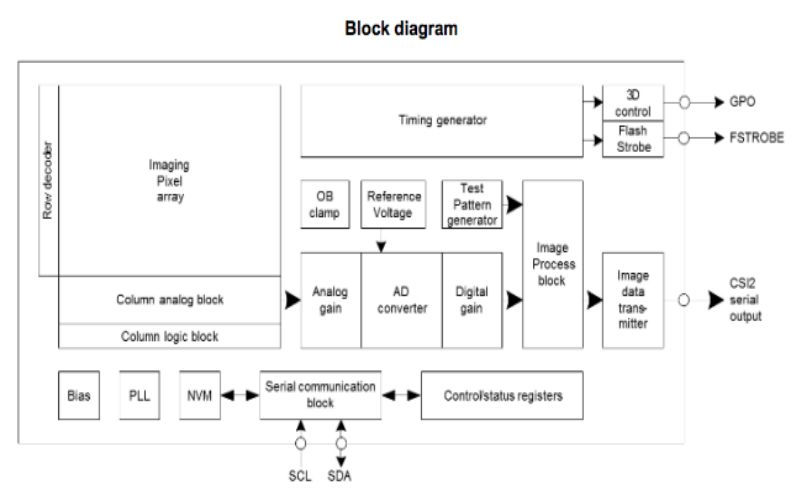

Figure 4. Sony IMX219 block diagram

\subsection{Optical adaptor}

For the optical adapter we used an FMA037 adaptor Figure 5 that is popular and largely used in the microscopic camera industry, the adapter is made for a $1 / 4 " \sim 1 / 3$ " sensor and is perfect for the sensor that we use in this application. The optical adaptor has a fixed focal length, $0.37 \mathrm{x}$ magnification and has the standard cmount to dia. $31.75 \mathrm{~mm}$ eye tube that is largely used in most microscopes.

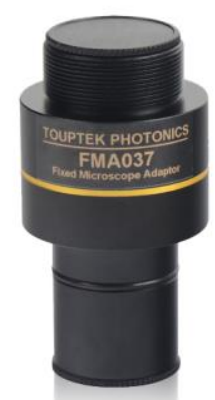

Figure 5. FMA037 Optical adapter

\subsection{LiPo Battery}

The camera uses a LiPo battery Figure 6 that provides enough power for about 8 hours of use, it has $5 \mathrm{~V}$ and $5500 \mathrm{mAh}$.

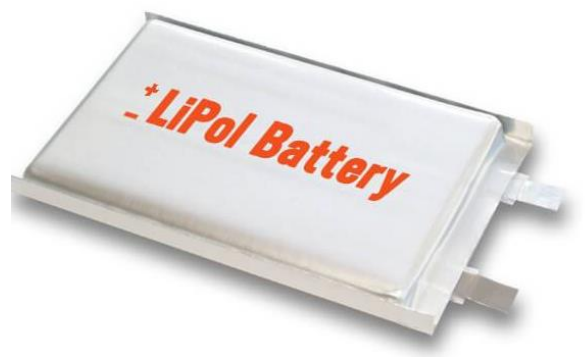

Figure 6. LiPo Battery

\section{WIRELESS CAMERA}

In Figure 7 and Figure 8 we have the commercial concept of the camera, the enclosure is made of aluminium, forming a unitary system that allows to take, wireless, photo/video of the image provided by the microscope (Figure 9). The photo/video is either send over IP/Web Interface or saved on the internal memory of the camera to be downloaded later [6].

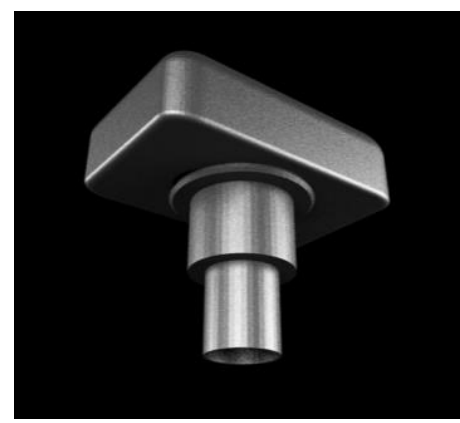

Figure 7. Commercial concept 1

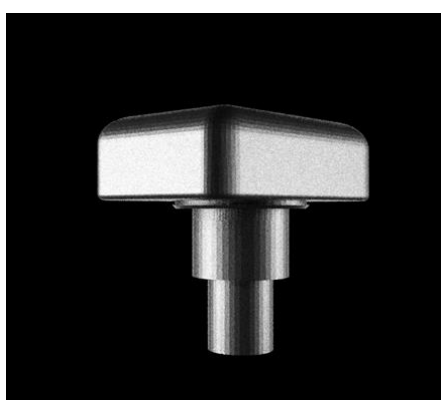

Figure 8. Commercial concept 2

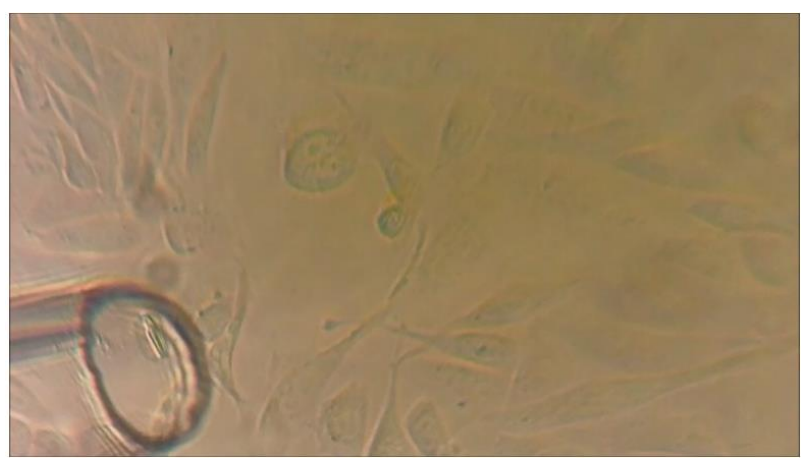

Figure 9. Image taken with the wireless camera

\section{CAMERA WEB INTERFACE}

The web interface that is used for our application is made in PHP, JS, CSS, Cam APK and works with the Raspberry camera, the interface can be used in different applications like surveillance, recording and time lapse photography. The user can access the camera from every web browser and It is highly configurable [7].

In Figure 10 we have functions like record video, record image, time lapse and motion detection, the user can view the live footage directly from the web browser all around the globe provided that he must have a good internet connection. 


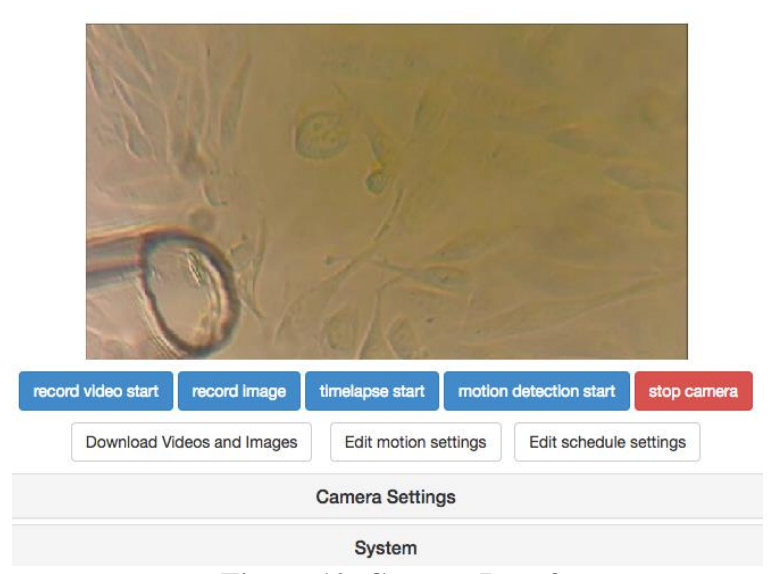

Figure 10. Camera Interface

In Figure 11 we have a few of setting for the camera. The settings are:

- Resolutions (the user can choose custom resolutions, fps for both video and photo);

- Time lapse interval;

- Annotation (to be implemented in the video/photo like a watermark with text and colour settings);

- Sharpness, Contrast and Brightness etc.

\begin{tabular}{|c|c|}
\hline \multicolumn{2}{|c|}{ Camera Setting $\underline{s}$} \\
\hline Resolutions: & $\begin{array}{l}\text { Load Preset: } \\
\text { Custom Values: } \\
\text { Video res: } \\
\text { Vidion... } \\
\text { Video fps: } 25 \text { recording, } 25 \text { boxing } \\
\text { Image res: } 2592 \times 1944 \mathrm{px} \\
\text { OK }\end{array}$ \\
\hline Timelapse-Interval (0.1...3200): & $3 \mathrm{~s} \mathrm{OK}$ \\
\hline Video Split (seconds, default $0=$ off): & $0 \mathrm{~s} O \mathrm{OK}$ \\
\hline Annotation (max 127 characters): & $\begin{array}{l}\text { Text: Camera Wireless } \text { OK Default } \\
\text { Background: Off } \mathbf{v}\end{array}$ \\
\hline Annotation size(0-99): & $50 \mathrm{OK}$ \\
\hline Custom text color: & Disabled y:u:v $=255: 128: 128$ OK \\
\hline Custom background color: & Disabled $\mathrm{y}: \mathrm{u}: \mathrm{v}=0: 128: 128 \mathrm{OK}$ \\
\hline Buffer (1000... ms), default 0 : & $0 \quad \mathrm{OK}$ \\
\hline Sharpness $(-100 \ldots 100)$, default 0 : & $0 \quad \mathrm{OK}$ \\
\hline Contrast $(-100 \ldots 100)$, default 0 : & $0 \quad \mathrm{OK}$ \\
\hline Brightness $(0 . .100)$, default 50 : & 50 OK \\
\hline
\end{tabular}

Figure 11. Camera Settings

The user can set the camera very easy from the web browser, the settings are vast in number and can apply for different applications.

In Figure 12 we have the system settings for the camera. The settings include functions like shutdown, reboot and reset. We can load different macros and give different functions for the video/photo/time lapse modes.

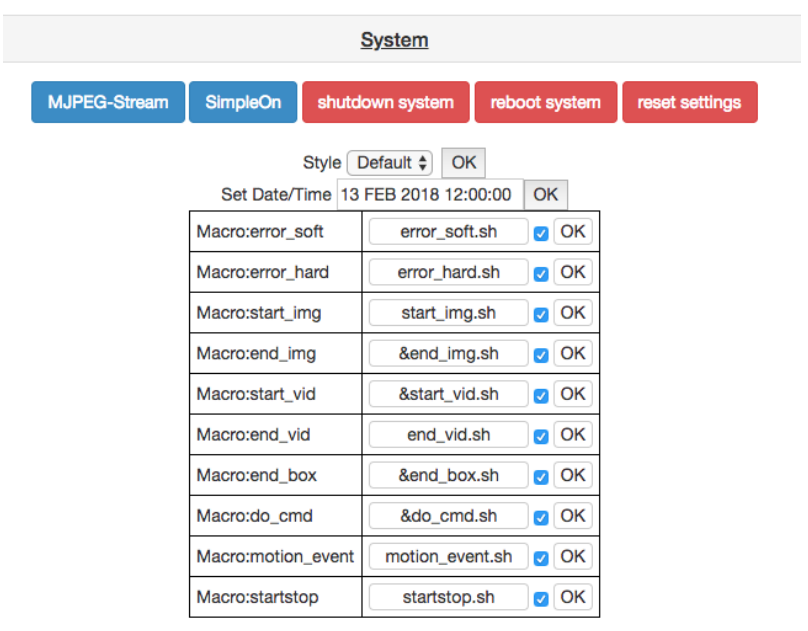

Figure 12. System Settings

\section{CONCLUSIONS}

The application is very useful and versatile and can be used in other fields for photo/video recording, time lapse and surveillance with minor modifications. It's highly configurable and makes a good economical choice without affecting performance to a microscope camera that we can find on the market but at a higher price. Also the application has wireless functionality.

\section{REFERENCES}

[1] F. Rost, and R. Oldfield, Photography with a microscope, Cambridge University Press, 2000.

[2] K. Kearney, and W. Freeman, Creative Projects with Raspberry Pi, Raspberry Pi Foundation, 2017.

[3] R. Nixon, Learning PHP, MySQL \& JavaScript: with jQuery, CSS \& HTML5, O’Reilly Ed., 2014.

[4] D. Singh, Fundamentals of Optics, PHI Learning Pvt. Ltd, 2015.

[5] D. Sklar, and A. Trachtenberg, PHP Cookbook, O'Reilly Ed., 2017.

[6] K, Yank, PPHP \& MySQL: Novice to Ninja: The Easy Way to Build Your Own Database Driven Website, SitePoint Pty. Ltd., 2012.

[7] G. R. Fowles, Introduction to modern optics, Dover Publications Inc., 1975. 